\title{
Enfisema subcutáneo facial autoinducido con la finalidad de conseguir deformidad facial y evitar reconocimiento legal
}

\section{Self-induced facial subcutaneous emphysema with the achieving facial deformity and prevent legal recognition}

\section{Resumen}

El enfisema facial subcutáneo se presenta en ocasiones en el marco de procedimientos odontológicos, traumatismos 0 cirugía. No es habitual que este enfisema sea auto-provocado por el paciente. Se informan tres casos de enfisema facial autoprovocado en reclusos con la intención de deformar la cara y evitar ser reconocidos por testigos en el juzgado.

Palabras clave: Lesiones autoprovocadas. Enfisema subcutáneo facial. Prisión. Sanidad penitenciaria.

\section{Abstract}

Facial subcutaneous emphysema may occur in the context of dental procedures, trauma or surgery. It is unusual that this emphysema is self-caused by the patient. We report three cases of facial emphysema produced by inmates in the prison with intent to disfigure the face and avoid being recognized by witnesses in court.

Key words: Self-induced injury. Emphysema subcutaneous facial. Penitentiary Health.

\section{Introducción}

La mayor parte de los casos de enfisema facial subcutáneo son consecuencia de complicaciones en procedimientos dentales ${ }^{1-5}$, traumatismos craneoencefálicos con fractura ósea ${ }^{6,7}$ y cirugía de cabeza y cuello ${ }^{8}$. Con menor frecuencia, la bibliografía describe enfisemas inducidos por el propio paciente involuntariamente, por mordedura ${ }^{9}$, vómito ${ }^{10}$, accidentes laborales entre músicos de instrumentos de viento o sopladores de vidrio ${ }^{11}$. En grado minoritario, hallamos casos facticios producidos de forma deliberada por el propio paciente; hecho que se describe en enfermos psiquiátricos ${ }^{12,13}$ y apenas hay referencias en los que destaque el carácter finalista de la autolesión, si bien, hay alguno descrito en población reclusa ${ }^{14,15}$.

El objetivo del presente estudio es analizar la casuística, procedimiento y finalidad del enfisema subcutáneo facial autoinducido en un centro penitenciario de jóvenes.

\section{Material y métodos}

Se ha valorado en los servicios médicos del centro penitenciario de jóvenes de Barcelona, en el año 2010, a los tres sujetos que se autoprovocaron un enfisema subcutáneo facial con el fin de deformar la cara y evitar la identificación en rueda de reconocimiento por parte de testigos. No tenemos evidencia de que se hayan producido otros casos similares. Se debe puntualizar que se desconoce la incidencia real de esta práctica, ya que los reclusos evitan que la autolesión sea conocida, y esta información sólo trasciende de manera excepcional.

Los tres reclusos eran varones, jóvenes, concretamente dos de 18 años y uno de 20 años de edad, de raza blanca y nacionalidad chilena. No padecían antecedentes patológicos de interés (somáticos o psiquiátricos) y no habían sido atendidos por autolesiones previas en la prisión.

\author{
AF. Bedoya ${ }^{1}$ \\ MJ. Leal ${ }^{1}$ \\ N. Lleopart $^{1}$ \\ PA. Martínez-Carpio ${ }^{1}$ \\ MT. Marrón ${ }^{2}$
}

${ }^{1}$ Médicos del Centro Penitenciario de Jóvenes Barcelona. ${ }^{2}$ Instituto de Medicina Legal de Cataluña.

Correspondencia: Bedoya AF

Ctra. de Masnou a Granollers Km 13.215

08026 La Roca del Vallès (Barcelona)

E-mail:

abedoyac@gencat.cat

Fecha de recepción: 16.JUN.2011

Fecha de aceptación: 21.JUL.2011 
El procedimiento utilizado consistió en practicarse, antes de la salida de prisión hacia la rueda de reconocimiento que tiene lugar en el juzgado, dos pequeñas perforaciones en la mucosa de la fosa canina superior, mediante punción con aguja de coser. Posteriormente, se insufla aire a través de las perforaciones mediante repetición de la maniobra de Valsava. Con la cavidad oral llena de aire a presión, éste se infiltra en el tejido submucoso y subcutáneo, acumulándose en las zonas de mayor laxitud (mejillas y párpados). El aire acumulado se presenta como una hinchazón de formación súbita, indolora, elástica y crepitante, que fácilmente se desplaza por los tejidos ante la presión digital. Una vez finalizada la rueda de reconocimiento, se realiza el drenaje del aire por el mismo punto de entrada, haciendo presión de la cara con las manos.

En ninguno de los casos descritos se produjeron complicaciones y, en un periodo de entre 2 y 4 días desapareció el enfisema. Los enfisemas se observaron en consulta, a la que acudieron los pacientes por tres motivos: a) A iniciativa del recluso, por no resolverse la deformidad mediante maniobras de autodrenaje; b) A instancias del personal de vigilancia que observó el enfisema y sospechó que había sido víctima de una agresión; c) A iniciativa del recluso que acudió por otro motivo y el médico lo detectó.

La práctica, según lo relatado por los reclusos, fue exitosa y evitó el reconocimiento, incluso, uno de ellos comentó que había repetido la maniobra en varias ocasiones.

\section{Discusión}

El diagnóstico diferencial se debe realizar con hematoma y edemas, principalmente edema angioneurótico.

Las posibles complicaciones que pueden aparecer son la de difusión del aire a la zona cervical y mediastino y la infección. Ante esta segunda posibilidad, debe indicarse la cobertura antibiótica del paciente. En nuestra casuística no se dio ninguna complicación ni se requirió tratamiento.

La asistencia médica en estos casos conlleva importantes implicaciones médicolegales, debiendo tener en cuenta el marco jurídico, el principio de proporcionalidad entre la defensa de la intimidad del paciente y la defensa de otros bienes de interés público. Hay circunstancias por las que estamos obligados a no guardar el secreto profesional: cuando constituya una medida necesaria para la seguridad pública, la prevención de infracciones penales, la protección de la salud pública o la protección de los derechos y libertades de las demás personas.

En España y en la mayoría de los países, el médico está obligado a poner en conocimiento de la Autoridad Judicial todo hecho originario de una lesión, en el amplio sentido de la palabra, de potencial etiología criminal, por escrito mediante el parte correspondiente.

En estos casos el procedimiento a seguir es realizar el parte de lesiones que en el medio penitenciario se eleva al director del centro para remitirlo al Juzgado competente.

La finalidad de la autolesión en nuestros pacientes fue distorsionar su imagen para impedir su reconocimiento. Una referencia de este tipo es el caso de un recluso alemán que se provocó las lesiones con la finalidad de conseguir un ingreso hospitalario ${ }^{14}$; otra referencia es la de cuatro sujetos de un mismo centro penitenciario, quienes se provocaron un enfisema cervical que fue seguido de neumomediastino, suponiéndose que la finalidad de la autolesión era forzar la asistencia sanitaria extrahospitalaria para su fuga ${ }^{15}$.

\section{Conclusión}

A nuestro criterio no existen muchos casos como los descritos, por lo que se aporta una información relevante a tener en cuenta, especialmente en el contexto médico-legal, judicial y en el ámbito penitenciario. 


\section{Bibliografía}

1. Pynn BR, Amato D, Walker DA. Subcutaneous emphysema following dental treatment: a report of two cases and review of the literature. J Can Dent Assoc. 1992;58(6):496-9.

2. Snyder MB, Rosenberg ES. Subcutaneous emphysema during periodontal surgery: report of a case. J Periodontol. 1977;48(12):790-1.

3. Uehara M, Okumura T, Asahina I. Subcutaneous cervical emphysema induced by a dental air syringe: a case report. Int Dent J. 2007;57(4):286-8.

4. Steelman RJ, Johannes PW. Subcutaneous emphysema during restorative dentistry. Int J Paediatr Dent. 2007;17(3):228-9.

5. Chen SC, LinFY, Chang KJ. Subcutaneous emphysema and pneumomediastinum after dental extraction. Am J Emerg Med. 1999;17(7):678-80.

6. Brasileiro BF, et al. Traumatic subcutaneous emphysema of the face associated with paranasal sinus fractures: a prospective study. J Oral Maxillofac Surg. 2005;63(8):1080-7.

7. Gottlieb O. Subcutaneous emphysema in fracture of facial bones. J Am Dent Assoc. 1967;75(1):167-9.

8. Shine NP, Sader C, Coates H. Cervicofacial amphysema and pneumomediastinum following pediatreic andenotosilectomy: a rare complication. Int J Pediatr Otorhinolaryngol. 2005;69(11):1579-82.

9. Yamada $\mathrm{H}$, et al. Facial emphysema caused by cheek bite. Int J Oral Maxillofac Surg. 2006;35(2):188-9. Epub 2005 Nov 8.

10. Siddiq MA, et al. Subcutaneous cervical emphysema after self-induced vomiting. J $R$ Soc Med. 1999;92:192-3

11. Han S, Isaasson G. Recurrent pneumoparotid: cause and treatment. Otolaryngol Head Neck Surg. 2004;131(5):758-61.

12. Tosun F, et al. Patient of severe cervicofacial subcutaneous emphysema associated with Munchausen's syndrome. J Craniofac Surg. 2005;16(4):661-4.

13. Alfio $F$, et al. Non-occupational recurrent bilateral pneumoparotitis in an adolescent. The Journal of Laryngology \& Otology. 1992;106:558-60.

14. Yattara-Baratti CL, Hoffmann R, Langlotz M. Softtissue cervicofacial emphysema and pneumomediastinum caused by self injury. Schweiz Med Wochenschr. 1988;118(31-32):1135-7.

15. López-Peláez MF, Roldán J, Mateo S. Cervical emphysema, pneumomediastinum, and pneumothorax following self-induced oral injury: report of four cases and review of the literature. Chest. 2001;120(1):306-9. 